\title{
Electronically Tunable Wide Band Optical Delay Line Based on InGaAs Quantum Well Microresonators
}

\author{
Yan Zhang ${ }^{1}$ and Geoff W. Taylor ${ }^{2}$ \\ ${ }^{1}$ ODIS Inc., 54 Ahern Lane, Storrs, CT 06269, USA \\ ${ }^{2}$ Department of Electrical \& Computer Engineering, University of Connecticut, 54 Ahern Lane, Storrs, CT 06269, USA
}

Correspondence should be addressed to Yan Zhang; yaz@engr.uconn.edu

Received 4 June 2013; Accepted 3 August 2013

Academic Editor: Armin Gerhard Aberle

Copyright (C) 2013 Y. Zhang and G. W. Taylor. This is an open access article distributed under the Creative Commons Attribution License, which permits unrestricted use, distribution, and reproduction in any medium, provided the original work is properly cited.

A novel electronically tunable optical delay line based on InGaAs quantum well microresonators is proposed for high frequency $\mathrm{RF}$ transmission. The device utilizes the charge-controlled blue shift of the absorption edge in InGaAs quantum wells to change the effective refractive indices of the resonators and couplers, therefore, provides an efficient way to produce variable time delay. A theoretical model based on measurements is used to analyze the device performance. Simulation results for five $3 \times 27 \mu \mathrm{m}^{2}$ cascaded resonators with bias voltages $<0.7 \mathrm{~V}$ show a continuous tuning range of $7 \sim 68 \mathrm{ps}$, a ripple delay $<1.5 \mathrm{ps}$, and a useable bandwidth of $39.3 \mathrm{GHz}$.

\section{Introduction}

Optical delay is a valuable concept for a multitude of microwave and millimeter wave applications. However to be useful, the value of this delay should be controlled electronically, and this has remained a challenging problem for the various implementations. This problem falls under the general classification of slow light which refers to the ability to decrease the group velocity of an optical medium in order to control the delay. Various approaches have been reported such as SOI ring resonators [1], SOI gratings [2], and LPCVD ring resonator [3]. But the most interest has centered on semiconductor waveguides because of the prospect of a high degree of device integration and room temperature operation as described recently [4]. The potential applications lie in the areas of information processing, communications, and the generalized RF area of Microwave Photonics (MWP). However, in all of these areas the intrinsic limitations of slow light have prevented its incorporation in high bit rate applications. All the proposed and reported tunable optical delay lines utilize the thermo-optic effect and, therefore, require high operating voltages and large footprints. For example, in the MWP area, silicon-on-insulator (SOI) microring resonators have been used to achieve full phase tuning over $40 \mathrm{GHz}$ but the implementation employs temperature variations with microheaters to control the resonant frequency of the ring. The thermal response time in the ms range and the associated power dissipation are clearly limitations for the device applications.

In this paper, we introduce a new concept for the microresonator which enables direct electrical control of slow light. This is implemented by the field effect control of charge in the input waveguide and the resonator separately, which shifts the absorption edge, resulting in index changes predicted by Kramers-Kronig relations, and provides coupling and resonance control as discussed later. The microresonator has been reported previously as a high-speed modulator in the form of a rectangle which utilized corner reflectors and straight waveguides $[5,6]$. A top view and cross-sectional view of the resonator through $\mathrm{A}-\mathrm{A}^{\prime}$ are shown in Figure 1. The inputoutput waveguide is implemented with a unique zig-zag configuration that realizes layout symmetry between the input waveguide and the resonator waveguide resulting in optimum coupling. It has the added advantage of supporting the fundamental mode of the resonator and rejecting higher order modes. In addition to these desirable properties, the input waveguide, by running parallel to the resonator waveguide, creates a directional coupler with the special capability that 


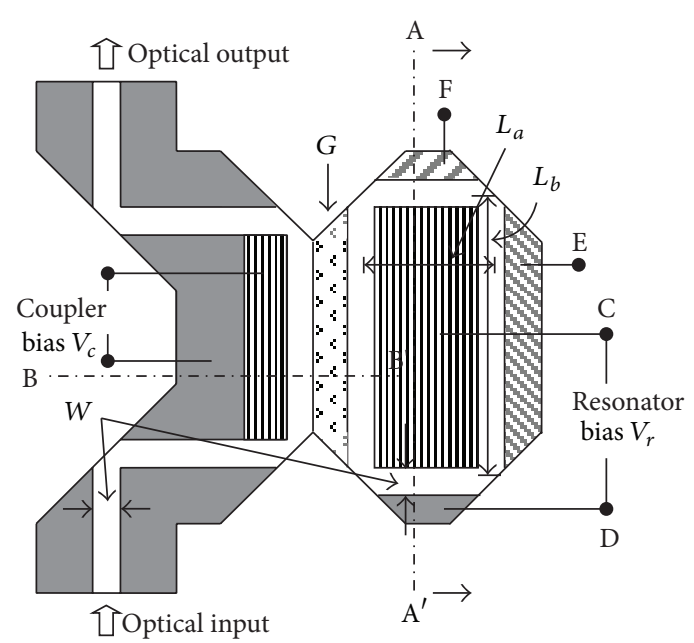

(a)

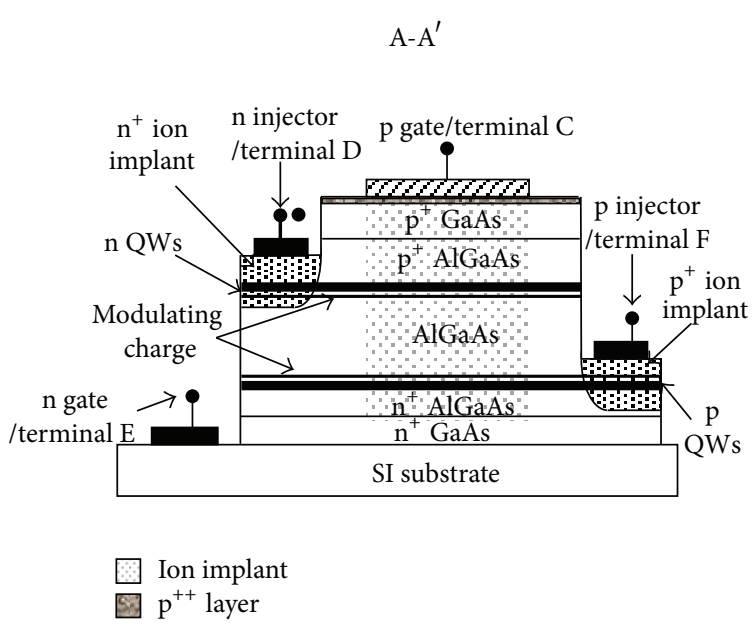

(b)

Figure 1: (a) Top view of the resonator/coupler. (b) A-A' cut view.

the charge may be injected into the input channel by electrical injection. This is indicated in Figure 1(b) by the coupler bias terminals and this enables the unique capability of voltage control of the coupling coefficient into the resonator [7]. Since the differential group delay of the resonator is controlled by the coupling coefficient, high-speed electrical control of slow light is, therefore, possible.

\section{Optical Time Delay Based on Microresonator Interactions}

The transfer characteristics of microresonators have been studied intensively $[8,9]$. The field transmission $T_{E}$ of the rectangular resonator is

$$
T_{E}=\frac{E_{\text {out }}}{E_{\text {in }}}=a \frac{\sqrt{1-K}-\sigma e^{-j \theta}}{1-\sigma \sqrt{1-K} e^{-j \theta}},
$$

where $a$ is the amplitude loss of the coupler, $K$ is the overall power coupling coefficient, and $\sigma=a \exp (-\Gamma \alpha L)$ is the amplitude loss per round trip in the resonator, where $L$ is the circumference of the resonator, $\Gamma$ is the confinement factor, $a$ is the absorption coefficient in the quantum wells, $\theta=$ $2 \pi L n_{\text {eff }} / \lambda$ is the phase shift per round trip, and $n_{\text {eff }}$ is the effective index at input wavelength $\lambda$. When a modulated optical carrier passes through a waveguide and interacts with the microresonator, a differential group delay is produced which is defined as

$$
\tau=-\frac{d \phi}{d \omega}=-\operatorname{Im}\left[\frac{1}{T_{E}} \frac{d T_{E}}{d \omega}\right]=\frac{\lambda^{2}}{2 \pi c} \operatorname{Im}\left[\frac{1}{T_{E}} \frac{d T_{E}}{d \lambda}\right],
$$

where $\phi$ is the phase angle of the field transmission in (1). Effectively the light has been delayed, thereby, creating slow light. It has also been well established [9] that when the loss is included the result is

$$
\tau=\frac{A(K)[C(K)-B(K) \cos \theta]}{[C(K)-B(K) \cos \theta]^{2}+E(K)^{2} \sin ^{2} \theta} \tau_{0},
$$

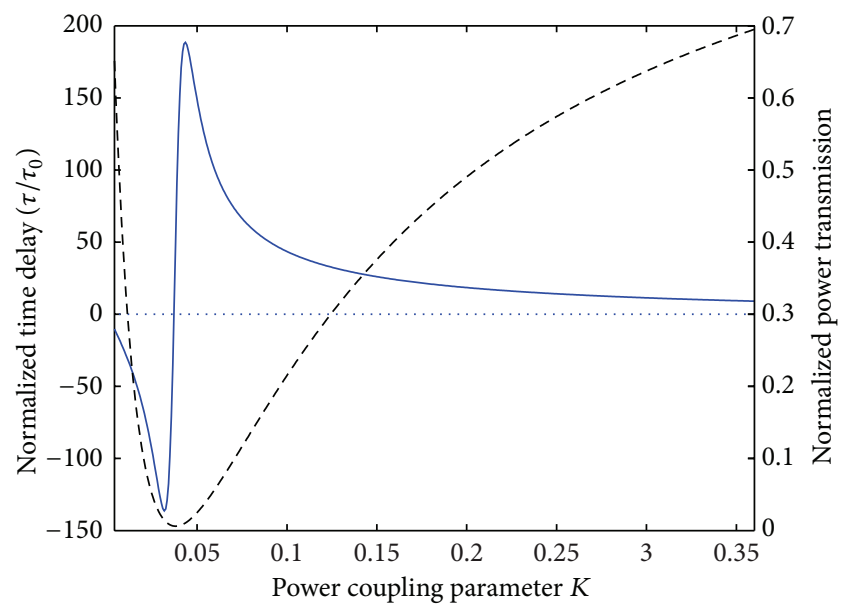

FIgURE 2: Normalized time delay (solid line) and transmission (dashed line) versus power coupling parameter $K$.

where $\tau_{0}=L n_{\text {eff }} / c$ is the resonator transit time, $c$ is the free space light velocity, and the functions $A(K) \equiv \sigma K, B(K) \equiv$ $\left(1+\sigma^{2}\right)(1+K)^{1 / 2}, C(K) \equiv \sigma(2-K), E(K) \equiv\left(1-\sigma^{2}\right)(1+K)^{1 / 2}$ all depend on the power coupling parameter $K$. For a lossless resonator, that is, $\sigma=1,(3)$ is reduced to

$$
\tau=\frac{K \tau_{0}}{2-K-2 \sqrt{1-K} \cos \theta} .
$$

The dependence of $\tau$ on coupling coefficient $K$ is illustrated in Figure 2 for $L=60 \mu \mathrm{m}, n_{\text {eff }}=3.3, \sigma=0.982$, and $\lambda=$ $1026.5 \mathrm{~nm}$, and it shows that as power coupling parameter $K$ is reduced very large values of delay are possible. The maximum delay is obtained near the minimum normalized transmission which is the condition of critical coupling in the resonator. It is also noted that large values of delay result from large values of $Q$ and, therefore, a very narrow frequency range over which the delay is realized. The group delay 


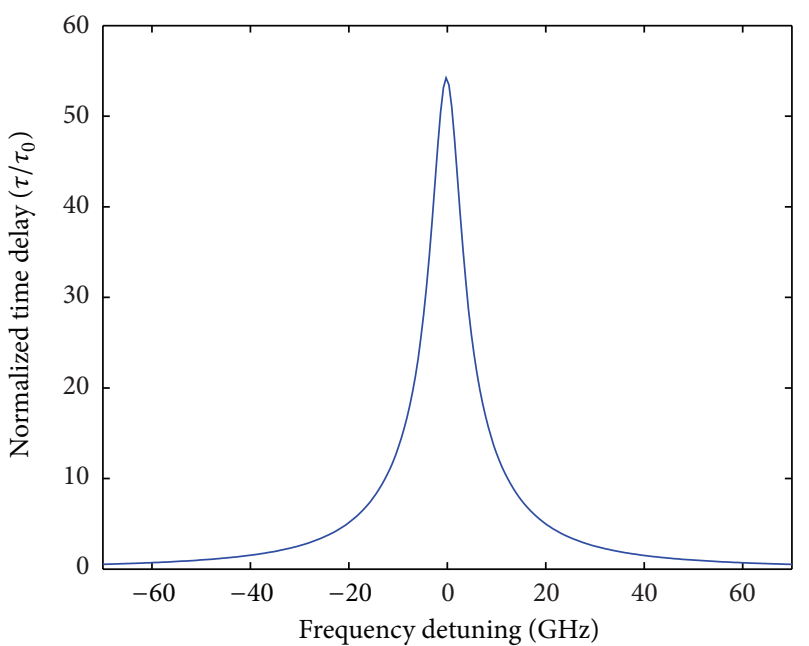

(a)

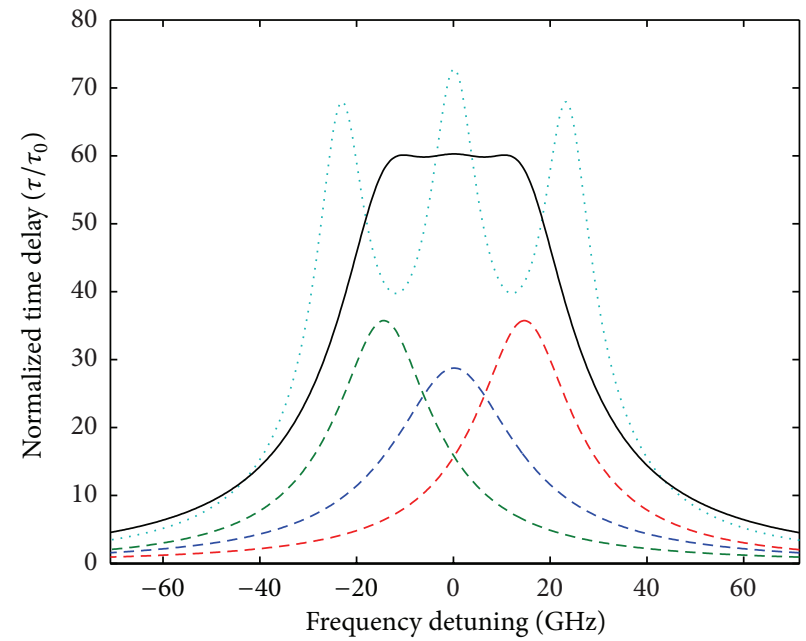

(b)

Figure 3: (a) Time delay spectrum for single resonator with $K=0.0785$. (b) Time delay spectrum of multiple resonators.

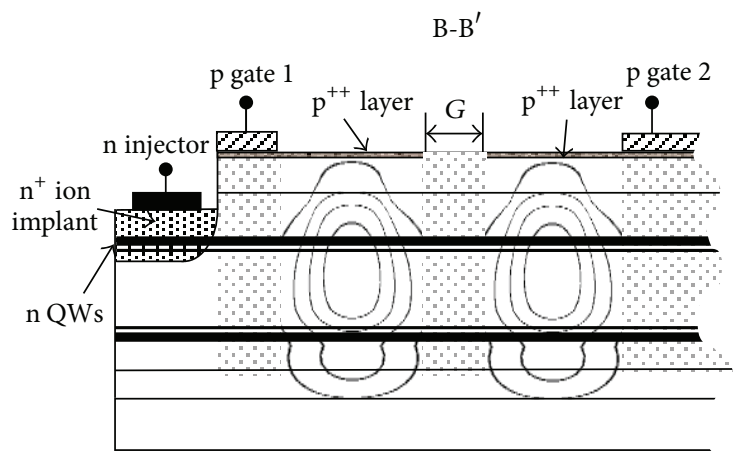

(a)

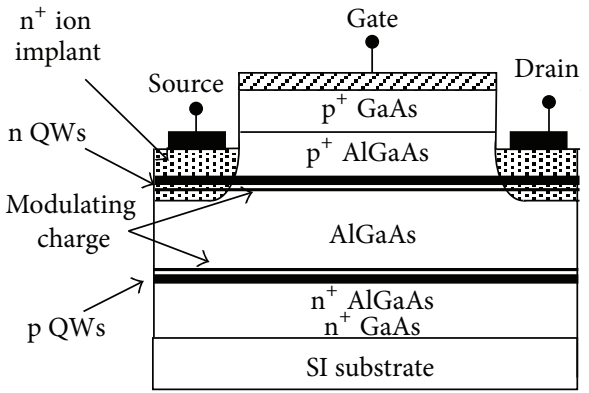

(b)

Figure 4: (a) B-B' cut view of the resonator/coupler. (b) Cut view of a transistor.

frequency response for single resonator with $K=0.0785$ is shown in Figure 3(a), which is centered at the resonant frequency of the resonator.

Clearly this is not the best choice because the narrow bandwidth of the ring resonator limits the transmitted frequencies. A natural solution is to use multiple resonators with different resonant frequencies so that the bandwidth of the time delay spectrum can be extended over a wider frequency range [10], as shown by the solid line in Figure 3(b) with individual resonator response shown by dotted lines. Note that for delay units consisting of multiple resonators, an inherent tradeoff exists between peak delay, bandwidth, delay ripple, and number of resonators. The time delay obtained with the three resonator contributions can still achieve a flattened bandpass region by optimizing the resonant frequency of each resonator and its power coupling $K$ to the waveguide. By comparison, the dotted line shows the result of using multiple resonators without proper adjustment. Since the top of the spectrum has larger ripples, RF transmission will suffer significant distortion.

\section{Device Structure and Characteristics}

As discussed, the key to a practical implementation of slow light is electrical control of both resonance frequency and $Q$ value of the resonators. This control is possible with the input waveguide and resonator combination shown in Figure 1. The structure is fabricated in the GaAs/InGaAs/AlGaAs system with a quantum well core in the waveguides as indicated in Figure 1. The quantum well is modulation doped with $n$ type doping just above the well. For the coupling control, we now consider the cross-section through B- ${ }^{\prime}$ in Figure 1(a) and this is shown in Figure 4(a). It is first noted that the waveguide is formed as a ridge with ion implants on either side. These implants are multifunctional. First, in the passive regions away from the resonator on both the input and output sides, they provide lateral optical confinement. However in the section $\mathrm{B}-\mathrm{B}^{\prime}$ that runs parallel to the resonator waveguide, the input waveguide is covered with a refractory metallization that masks the ion implant and creates the transistor structure. To appreciate this, the transistor structure with source 


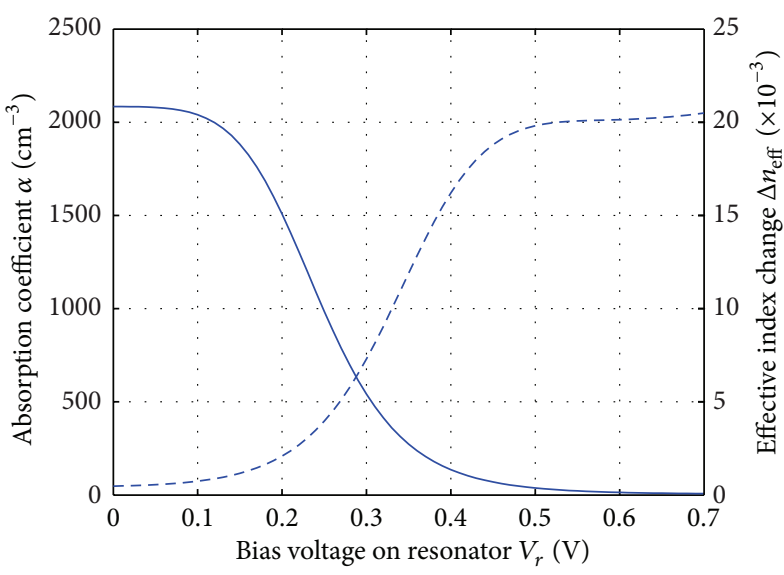

(a)

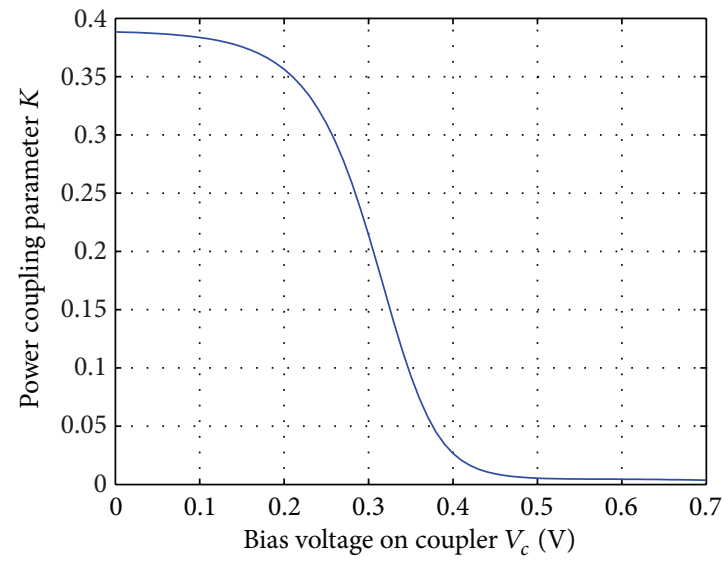

(b)

FIGURE 5: (a) Absorption coefficient (solid line) and effective index change of resonator (dashed line) versus bias voltage $V_{r}$ for $\lambda=1026.5 \mathrm{~nm}$. (b) Power coupling parameter $K$ versus bias voltage $V_{c}$ on coupler.

and drain contacts and a completely metalized gate region is shown in Figure 4(b). In Figure 4(a), since the surface is $p$ doped, then the gate voltage is still effectively applied when the optical aperture is formed by metal removal. Also, without the drain terminal the channel charge continues to respond to the changes in gate-to-source voltage.

The device shown in Figure 1 with $W=1 \mu \mathrm{m}, G=$ $1 \mu \mathrm{m}, L_{a}=3 \mu \mathrm{m}, L_{b}=27 \mu \mathrm{m}$, and an input wavelength of $1026.5 \mathrm{~nm}$ has been described [5] in terms of an analytical absorption model based upon the blue shift of the absorption edge. The absorption model has been combined with an electrical model for the transistor. Thus, when a bias voltage $V_{r}$ is applied to the resonator indicated in Figure 1(a), the absorption coefficient changes (and also $\sigma$ ) as shown in Figure 5(a) with the associated effective index change $\Delta n_{\text {eff }}$ in the resonator via Kramers-Kronig relations. Thus, the resonant frequency $v_{r}=c /\left(n_{\text {eff }} L\right)$ is modified. In addition, the absorption edge shift causes changes in the $\sigma$ value of the resonator. Simultaneously, another bias voltage $V_{c}$ (see Figure 1(a)) is applied to the waveguide arm of the coupler which realizes corresponding absorption and index changes in the input guide. Using RSOFT the decreases in coupling $K$ between the waveguide and the resonator are determined and plotted in Figure 5(b). The combined changes of $K$ and $\sigma$ reduce the $Q$ value of the resonator and, therefore, expand the bandwidth of the spectrum, while the change $\Delta n_{\text {eff }}$ adjusts the frequency of the resonance. Both of these dependences are important and in Figure 6, the combined effect of both bias voltages on the time delay spectrum of the resonator-coupler combination is shown (calculated using the design tool in Agilent's Advanced Design System).

It shows that combinations of $V_{r}$ and $V_{c}$ may be used to control the resonator characteristic and realize a wider possible time delay spectrum by trading off time delay amplitude for bandwidth. Then to maintain larger time delay amplitude together with enhanced bandwidth, multiple resonators are combined as discussed next.

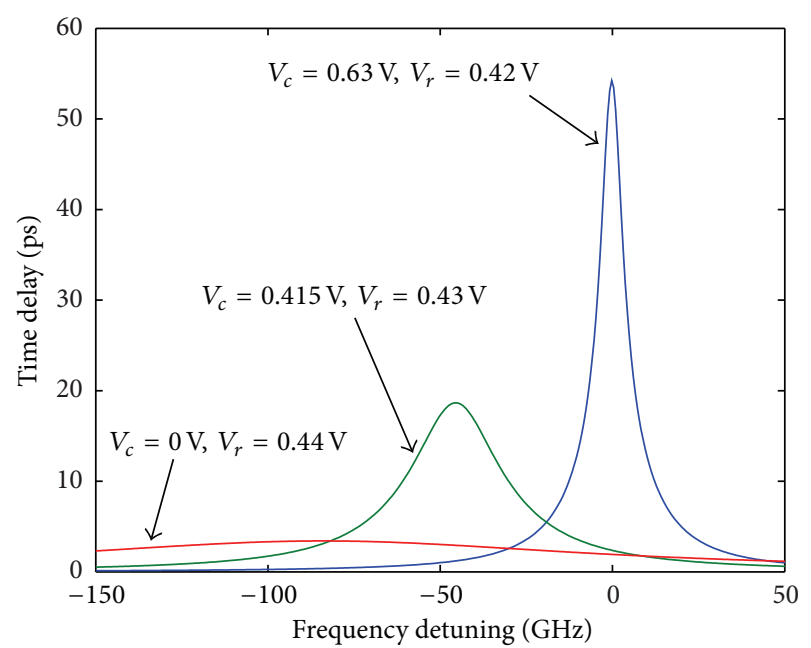

Figure 6: Time delay spectrum of ring resonator when different voltages are applied on the resonator and coupler.

\section{Optimization of Bandwidth and Time Delay}

To make use of slow light in a practical MWP application, the combined effects of resonator and coupler bias are used to adjust the parameters of multiple resonators in unique ways. A typical situation in which five resonators with $W=1 \mu \mathrm{m}$, $G=1 \mu \mathrm{m}, L_{a}=3 \mu \mathrm{m}, L_{b}=27 \mu \mathrm{m}$, and is arranged on one side of the waveguide in series with an optical amplifier which can also be integrated in the same circuit. Using the integrated format for the microresonators, there are unavoidable losses due to the minimum values of $\alpha$, such as the resonator corner loss and the coupler loss (typical values are $2.6 \mathrm{~dB}$ /resonator), and so it is expected that signal restoration will be required by the appropriate positioning of inwaveguide optical amplifiers. By individually adjusting the bias voltages on each resonator and coupler, a composite time 


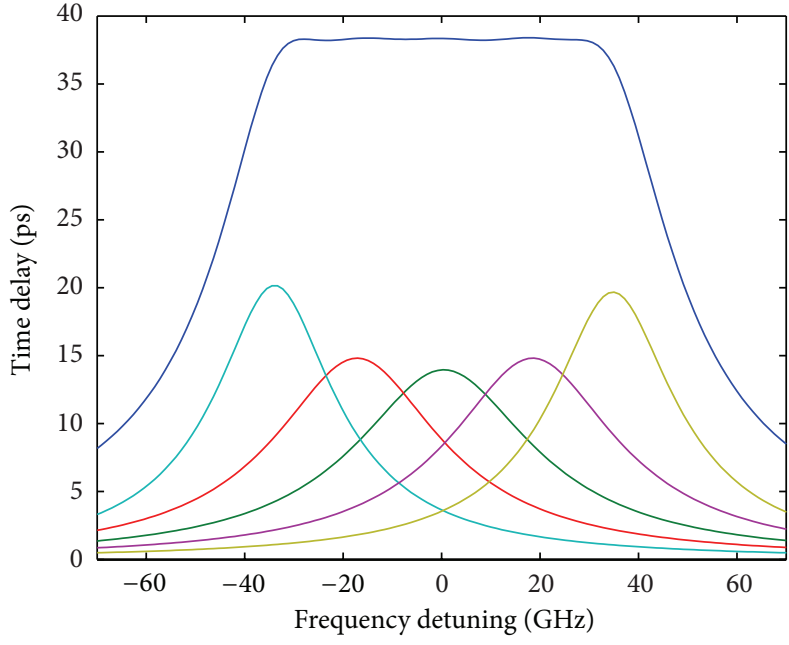

(a)

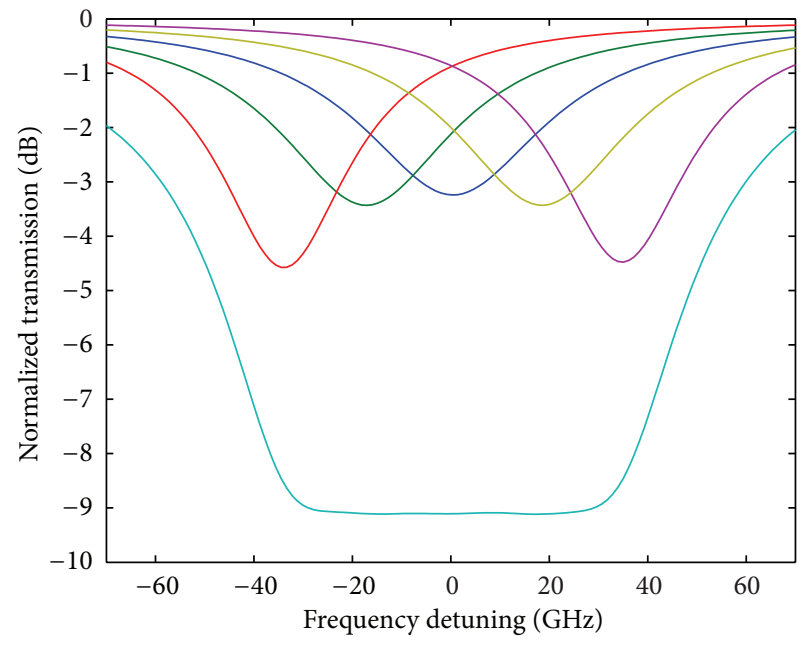

(b)

Figure 7: (a) Time delay spectrum of five resonators in series. (b) Transmission spectrum of five resonators in series without coupler loss included.

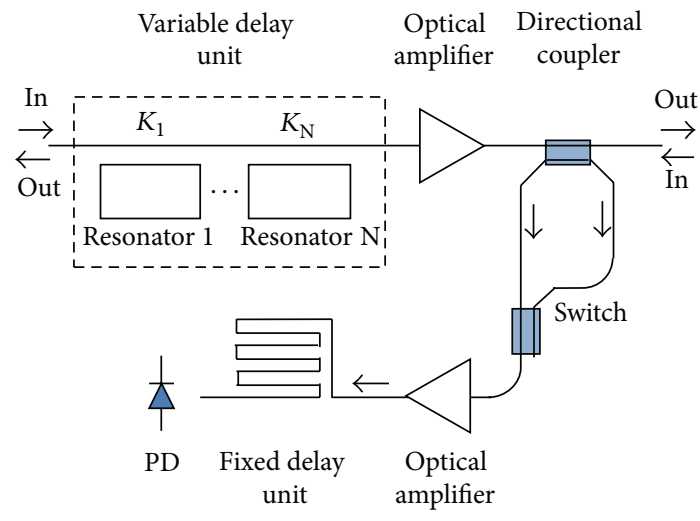

(a)

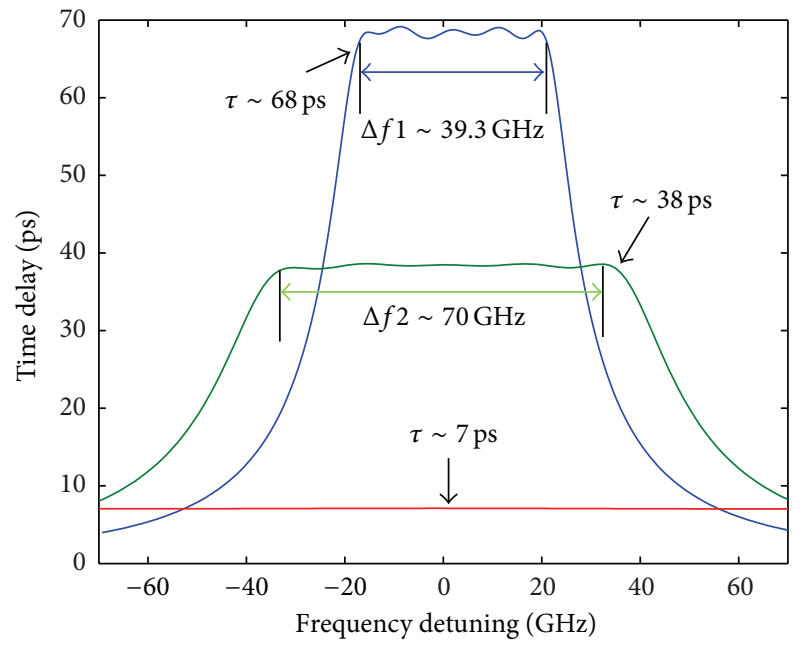

(b)

Figure 8: (a) Schematic of PAA delay unit. (b) Frequency spectrum of time delay unit consisting of five resonators under different configuration.

delay spectrum with 38 ps delay and about $70 \mathrm{GHz}$ useable bandwidth is realizable as shown in Figure 7(a). The corresponding transmission spectrum is shown in Figure 7(b).

One important application of this multiple resonator system is the generation of time delay for beam steering in a phased array system. A schematic of a (periodic phased array) PAA delay unit is shown in Figure 8(a). For an RF carrier of $35 \mathrm{GHz}$ (Ka band), a time delay difference between elements (i.e., variable delay unit of three resonators) of $0.7 \mathrm{ps}$ at a minimum and $14 \mathrm{ps}$ at a maximum would allow continuous beam steering over an angle of $0^{\circ} \sim 90^{\circ}$ with a time spectrum width of $>40 \mathrm{GHz}$. With proper adjustment of $V_{r}$ and $V_{c}$, a variable delay unit consisting of five resonators can provide a time delay ranging from $7 \mathrm{ps}$ to $\sim 68 \mathrm{ps}$ with a ripple $<1.5 \mathrm{ps}$ and can allow transmission of RF signals up to $39.3 \mathrm{GHz}$, as shown in Figure 8(b). With the aid of a fixed delay unit and optical switches as shown in Figure 8(a), a $180^{\circ}$ steering angle can be achieved which would require a bidirectional optical feed as shown. It is evident that an optoelectronic circuit that provides electronics to perform the adjustment of, $\sigma$ (by the use of optical amplifiers) and $\Delta n_{\text {eff }}$ on a per resonator basis would be an ideal approach to achieve electronic scanning in both $x$ and $y$ directions.

\section{Conclusion}

In this paper, we have described a novel electronically tunable optical delay line based on InGaAs quantum well microresonators. Time delay units are comprised of multiple rectangular microresonators cascaded on one side of the 
waveguide. The resonant frequency of each resonator and the coupling between the resonators and the waveguide can be adjusted by electronically controlling the effective index of the resonators and couplers. The performance of time delay units consisting of five series resonators is evaluated in FDTD, MATLAB, and ADS software. A time delay ranging from $7 \mathrm{ps}$ to $\sim 68 \mathrm{ps}$ with a ripple $<1.5 \mathrm{ps}$ and enabling transmission of RF signals up to $39.3 \mathrm{GHz}$ is obtained. For RF signals with frequencies as high as $70 \mathrm{GHz}$, a time delay up to $40 \mathrm{ps}$ can be provided. Finally, a time delay unit appropriate for application in a PAA is presented as an example of the device.

\section{References}

[1] M. S. Rasras, C. K. Madsen, M. A. Cappuzzo et al., "Integrated resonance-enhanced variable optical delay lines," IEEE Photonics Technology Letters, vol. 17, no. 4, pp. 834-836, 2005.

[2] S. Khan, M. A. Baghban, and S. Fathpour, "Electronically tunable silicon photonic delay lines," Optics Express, vol. 19, no. 12, pp. 11780-11785, 2011.

[3] C. G. H. Roeloffzen, L. Zhuang, R. G. Heideman, A. Borreman, and W. van Etten, "Ring resonator-based tunable optical delay line in LPCVD waveguide technology," in Proceedings of the 10th Annual Symposium of the IEEE/LEOS Benelux Chapter, Mons, Belgium, 2005.

[4] M. Santagiustina, G. Eisenstein, L. Thevenaz et al., "Slow light devices and their applications to microwaves and photonics," IEEE Photonics Society News, vol. 27, pp. 5-12, 2012.

[5] Y. Zhang, B. Pile, and G. W. Taylor, "Design of microresonator quantum well intensity modulators based on an absorption blue-shift," in Nanophotonics and Macrophotonics for Space Environments V, Proceedings of SPIE, San Diego, Calif, USA, August 2011.

[6] Y. Zhang, B. Pile, and G. W. Taylor, "Design of micro resonator quantum well intensity modulator," Optical and Quantum Electronics, vol. 44, no. 14, pp. 635-648, 2012.

[7] Y. Zhang and G. W. Taylor, "Quantum well micro-resonator optical modulator based on electrically controlled coupling," Optics Communications, vol. 305, pp. 282-288, 2013.

[8] A. Yariv, "Universal relations for coupling of optical power between microresonators and dielectric waveguides," Electronics Letters, vol. 36, no. 4, pp. 321-322, 2000.

[9] O. Schwelb, “Transmission, group delay, and dispersion in single-ring optical resonators and add/drop filters-a tutorial overview," Journal of Lightwave Technology, vol. 22, no. 5, pp. 1380-1394, 2004.

[10] G. Lenz, B. J. Eggleton, C. K. Madsen, and R. E. Slusher, "Optical delay lines based on optical filters," IEEE Journal of Quantum Electronics, vol. 37, no. 4, pp. 525-532, 2001. 

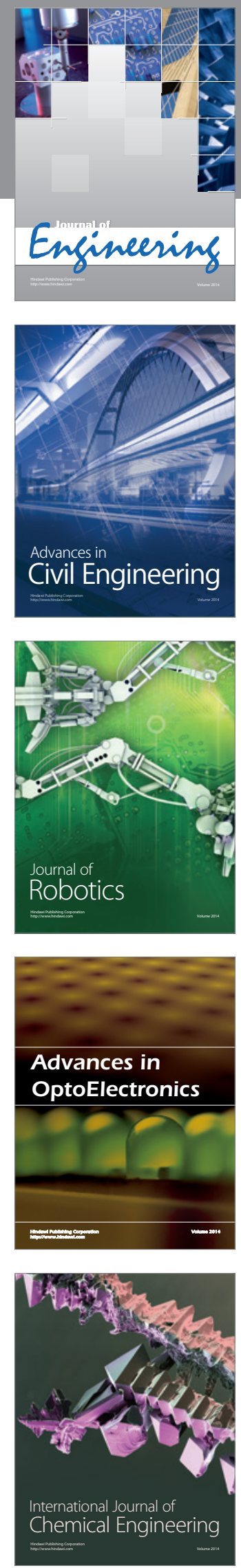

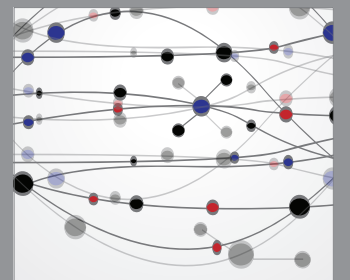

The Scientific World Journal
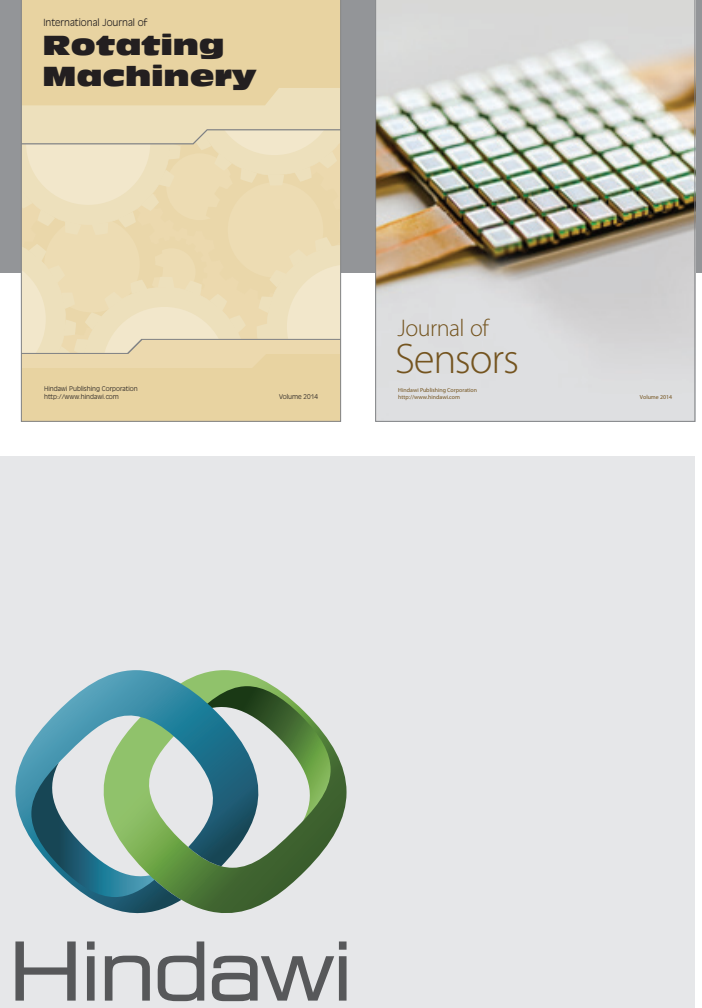

Submit your manuscripts at http://www.hindawi.com
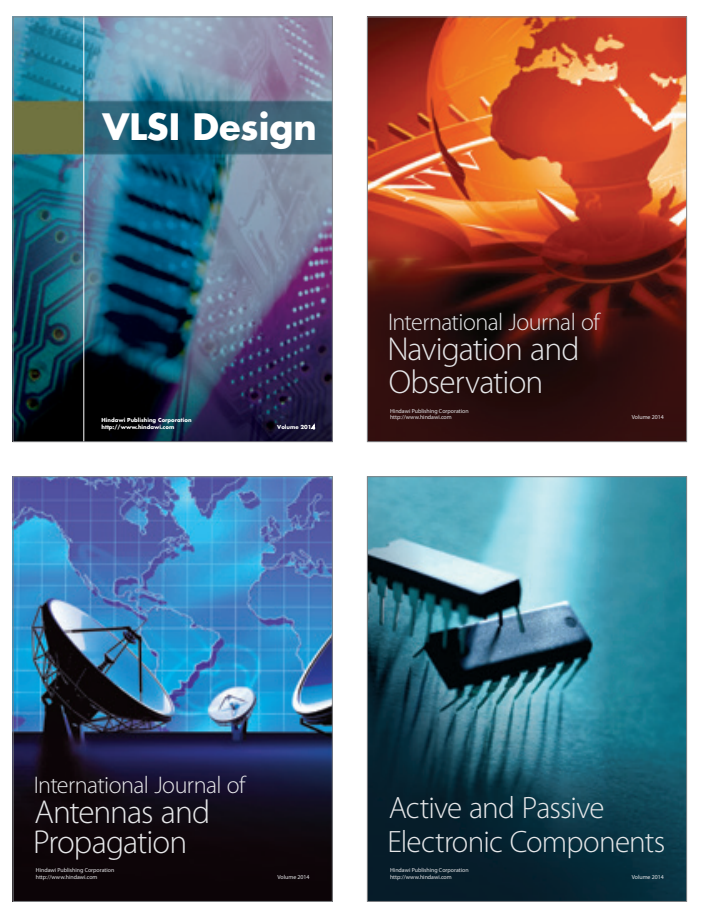
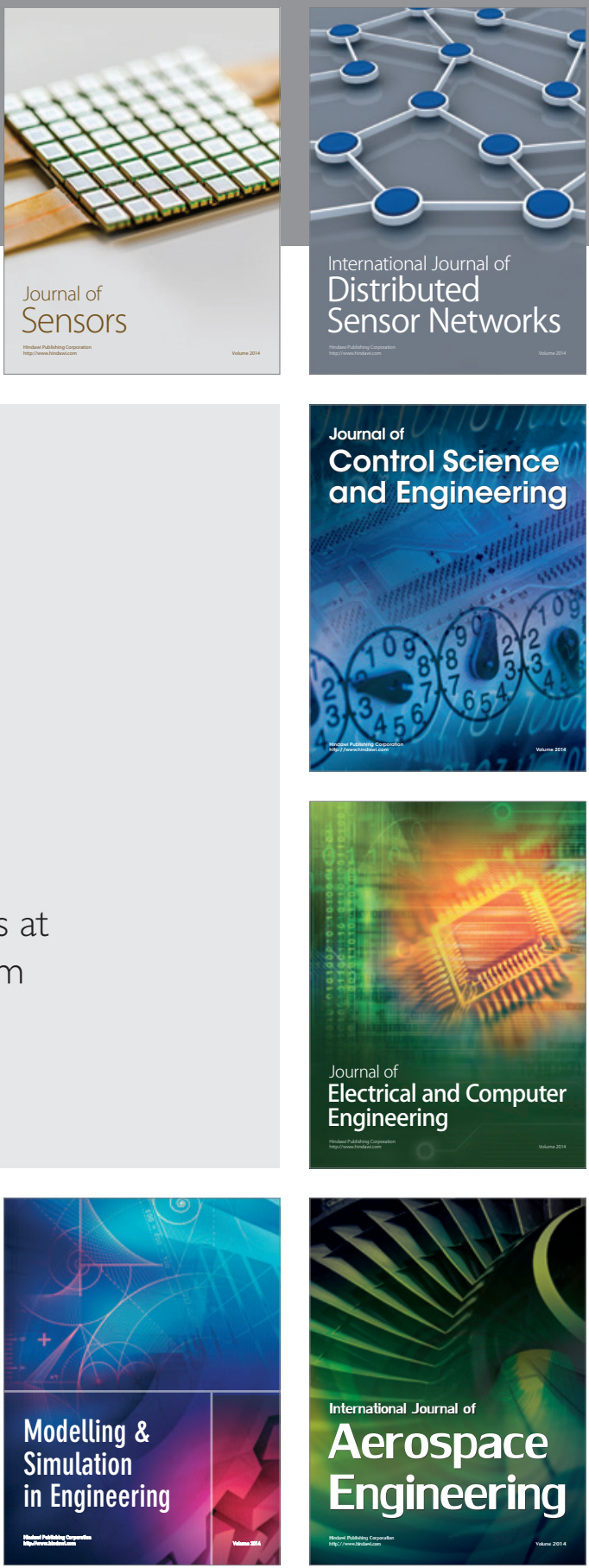

Journal of

Control Science

and Engineering
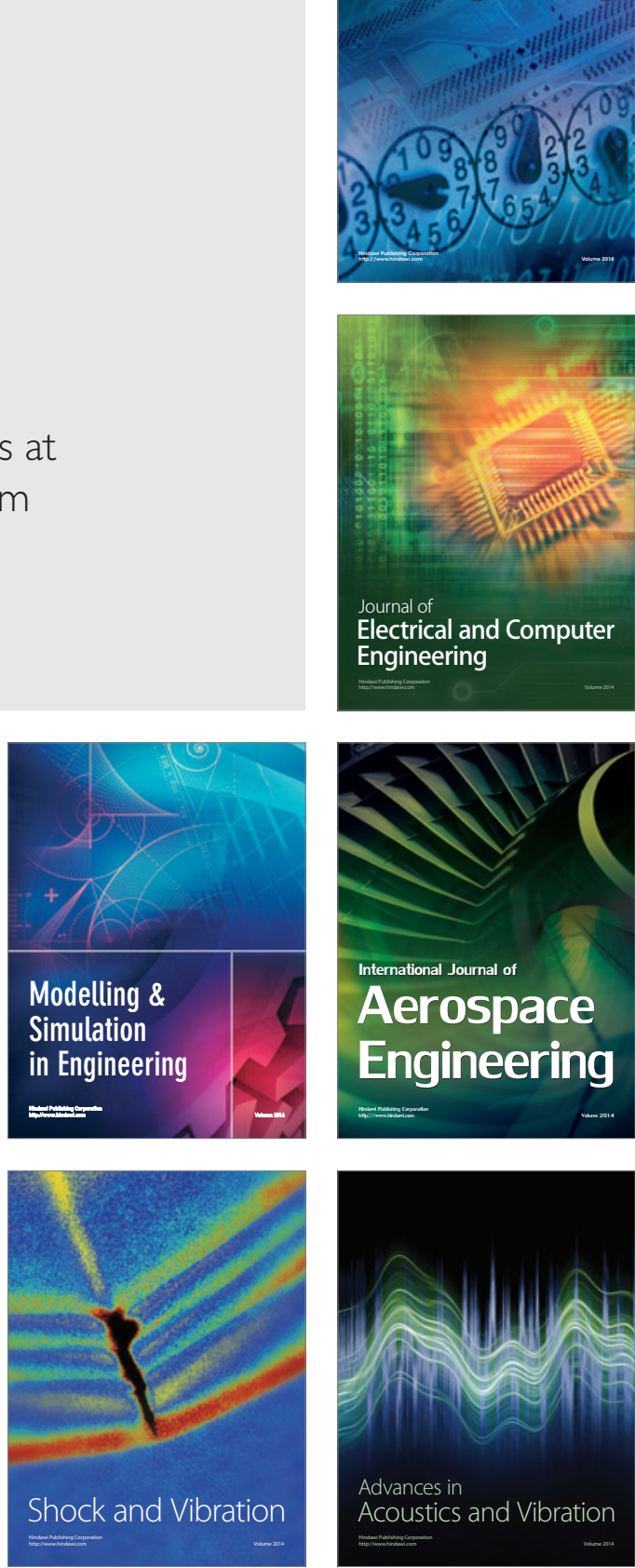